\title{
Optical Measurement of High-voltage Discharge in Air for Lidar Lightning Detection
}

\author{
Tatsuo SHIINA, ${ }^{1}$ Toshio HONDA, ${ }^{1}$ and Tetsuo FUKUCHI ${ }^{2}$ \\ ${ }^{1}$ Graduate school of Advanced Integration Science, Chiba University, 1-33 Yayoi-cho, Inage-ku, Chiba-shi, 263-8522 \\ ${ }^{2}$ Central Research Institute of Electric Power Industry, 2-6-1 Nagasaka, Yokosuka-shi, Kanagawa 240-0196
}

(Received February 4, 2008)

\begin{abstract}
This study aims to develop a new concept lidar for lightining strike prediction. In cloud-to-cloud discharges, the electromagnetic pulse rotates the polarization plane of a beam propagating beam through an ionized atmosphere due to the Faraday effect. Although this effect is small for visible light, it can be detected by a lidar system with an extinction ratio of polarization of $>30 \mathrm{~dB}$. In this study, the rotation of the polarization plane of a propagating beam due to the Faraday effect was detected in a high-voltage discharge experiment and verified by the numerical analysis results of the cloud-to-ground returning strike model. In the experiment, the shock wave due to the discharge was detected in addition to the rotation of the polarization plane. Based on these results, a scenario for cloud-to-cloud discharge detection by lidar measurement is proposed, and is reflected in lidar development.
\end{abstract}

Key Words: Faraday effect, Lightning, Discharge, Polarization, Lidar

\section{Introduction}

Lightning discharge detection using radio antennas requires plural observation points and synchronous action of the antennas. Radar works as stand alone system, while its use is limited due to legal restrictions on the usage of radio waves. Although observation of lightning from satellites is effective for global positioning, the altitude of the discharge and the weather condition under the thunderclouds cannot be known.

If a lidar system can detect lightning phenomena, it can serve as a stand alone system without limitation due to legal restrictions on the usage of radio waves. Furthermore, the 3-dimensional positioning of the discharge is possible because of time-of-flight measurement. There is few papers which reported the lidar lightning measurement. ${ }^{1)}$ Correlation between the propagating beam and the electromagnetic pulse caused by discharge phenomena mainly appears as the Faraday effect. The electromagnetic pulse rotates the polarization plane of a beam propagating through an ionized atmosphere. Based on numerical analysis using a return stroke model of a cloudto-ground discharge, which was based on Uman's model, ${ }^{2-4)}$ the rotation angle of the polarization plane has been estimated within less than $1 \mathrm{deg} .{ }^{5)}$ The authors have experimentally verified these results by discharge experiments using a small chamber. ${ }^{6)}$

In this study, the dependence of the rotation angle on the discharge and the arrangement between the discharge path and the propagating beam were examined by high voltage discharge experiments in air. The polarity of the charge voltage (direction of the discharge current), the gap length between the electrodes, and the distance between the discharge path and the propagating beam are parameters which influence the magnitude of the Faraday effect.

\section{Principle}

The magneto-optical effect (Faraday effect) has been reported as an optical measurement method for magnetic confinement fusion reactor. ${ }^{7)}$ The polarization plane of a beam propagating parallel to the magnetic flux is rotated in an ionized atmosphere or plasma (Fig. 1). The rotation angle is proportional to the product of the ionization electron density $n_{e}$ and the magnetic flux density $B$ along the beam propagation path. The linearly polarized beam can be regarded as a combination of the clockwise and the counterclockwise circularly polarized beams. The refractive indices of the ionized atmosphere for each circularly polarized beam are as follows; ${ }^{8)}$

$$
\begin{aligned}
& n_{ \pm}=\left(1-\frac{\omega_{p e}^{2}}{\omega^{2}} \frac{\omega}{\omega \pm \omega_{c e}}\right)^{1 / 2} \\
& \omega_{p e}=\sqrt{\frac{e^{2} n_{e}}{\varepsilon_{0} m_{e}}} \quad \omega_{c e}=\frac{e B}{m_{e}}
\end{aligned}
$$

where $\omega_{p e}, \omega_{c e}$ are the plasma and electron cyclotron frequencies, respectively, $e$ is the fundamental charge, $m_{e}$ is the electron mass, and $\varepsilon_{0}$ is the permittivity of free space. Therefore, the polarization rotation angle $\delta$ of a beam which has propagated over a distance $L\left(=L_{2}-L_{1}\right)$ is obtained as follows;

$$
\begin{aligned}
\delta & =\frac{2 \pi}{\lambda} \int_{L_{1}}^{L_{2}}\left(n_{+}-n_{-}\right) d l \\
& =2.62 \times 10^{-13} \lambda^{2} \int_{L_{1}}^{L_{2}} n_{e} B d l
\end{aligned}
$$

where $\lambda$ is wavelength of the propagating beam. Since $\delta$ is proportional to $\lambda^{2}$, the rotation angle for visible light will be small. Therefore, the polarization rotation angle must be measured with high accuracy. From Eq.(2), it is not possible to separately determine $n_{e}$ and $B$. However, the difference in the 
temporal dependencies of $n_{e}$ and $B$ may be used to separate their contributions to $\delta$. ${ }^{5}$

When the Faraday effect is applied to lightning measurement, the atmosphere needs to be ionized, and the magnetic flux due to the lightning discharge must exist. Cloud-to-cloud discharges, which cause 20-30 continuous discharges, satisfy those conditions. In general, the ionization of the atmosphere due to a lightning discharge is nearly perfect $\left(n_{e} \sim 10^{25} \mathrm{~m}^{-3}\right)$, while the diameter of the discharge path is in the order of $\mathrm{cm}$. The ionized atmosphere will only exist near the discharge path.

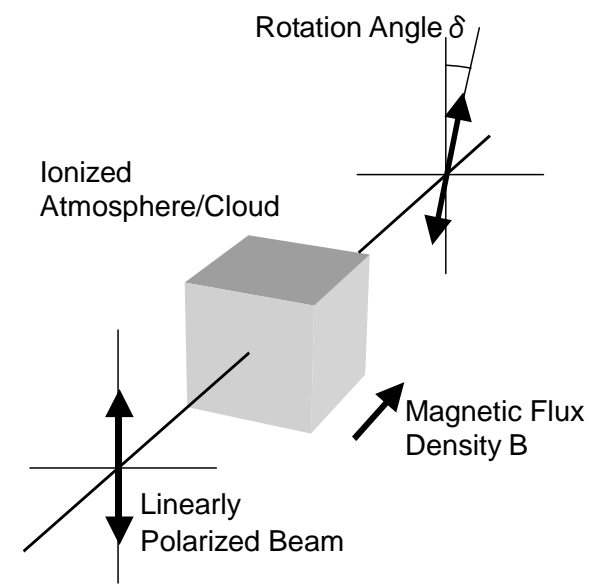

Fig.1 Schematic diagram of the Faraday effect.

\section{Experiment}

3.1 Experimental setup

Figure 2 shows the experimental setup of the high-voltage discharge experiment. The experiment was conducted in a high voltage experiment hall using an impulse voltage generator (HAEFELY SG $\triangle \mathrm{A} 1600-80$ ). The discharge gap between the needle electrodes was $0-2 \mathrm{~m}$ and the charging voltage was $>1000 \mathrm{kV}$. A voltage divider and Rogowski coil were used to measure the charge voltage and the discharge current. The laser beam was transmitted near the discharge path. The polarization plane of the linearly polarized laser beam was so adjusted by a half wave plate (HWP) that its photon flux was equally divided into the two mutually orthogonal polarization components. The intensities of the orthogonal polarization components were detected by photodiodes (PDs) with amplifiers. A differential amplifier was also installed in the receiver circuit to detect the small rotation angle of the polarization plane. To eliminate electromagnetic noise caused by the discharge, the laser power supply and the receiver circuit were placed inside copper boxes. Signal cables were also shielded by wire mesh. The specification of the discharge equipment and the optical detection system are summarized in Table 1 . The differential output was detected only when the polarization plane was rotated by the Faraday effect. The position of the propagating beam could be adjusted with respect to the discharge path and the discharge terminals.

The rotation angle of the polarization plane was estimated from the intensities of the orthogonal polarization components or the differential output by the equation;

$$
\begin{aligned}
\delta & =\frac{\pi}{4}-\tan ^{-1}\left(\sqrt{I_{s}} / \sqrt{I_{p}}\right) \\
& =\tan ^{-1}\left(\frac{\sqrt{I_{p}}-\sqrt{I_{S}}}{\sqrt{I_{P}}+\sqrt{I_{S}}}\right)
\end{aligned}
$$

$I_{p}$ and $I_{s}$ are the intensities of the orthogonal polarization components.

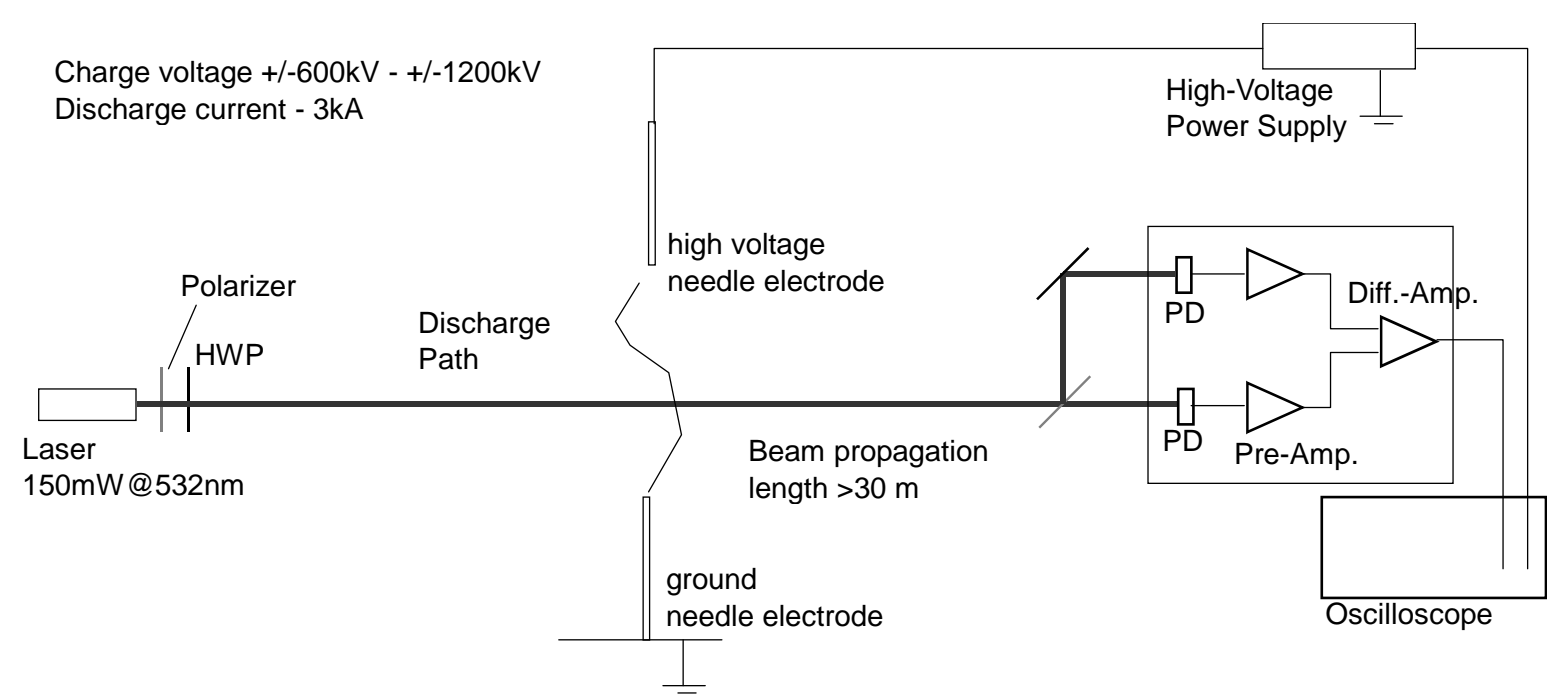

Fig.2 Experimental setup of the high voltage discharge experiment. 


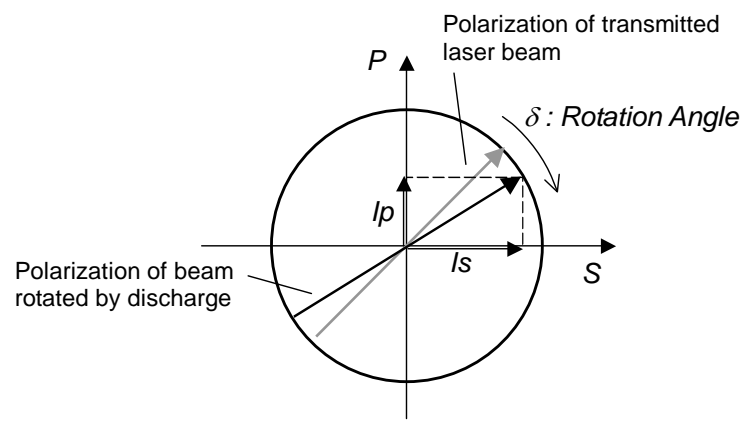

Fig.3 Differential detection.

Table 1 Specifications of the discharge equipment and the optical detection system

\begin{tabular}{|l|l|}
\hline \multicolumn{2}{|l|}{ Discharge equipment } \\
\hline Manufacturer, model & HAEFELY SG $\Delta$ A1600-80 \\
\hline $\begin{array}{c}\text { Maximum charging } \\
\text { voltage }\end{array}$ & $+/-1600 \mathrm{kV}$ \\
\hline Discharge waveform & Lightning Impulse \\
\hline Electrodes & needles \\
\hline Discharge gap length & $0-2 \mathrm{~m}$ \\
\hline Beam/Receiver & $\begin{array}{l}\text { Nd:YAG green laser } \\
\lambda=532 \text { nm, CW }\end{array}$ \\
\hline Light source & 150 mW \\
\hline Power & Photodiode + Amplifier \\
\hline Detector & Differential detection \\
\hline detection &
\end{tabular}

When $|I p-I s|<<$ Ip, Is, the rotation angle is approximated by the following equation;

$$
\delta=\frac{I_{p}-I_{s}}{I_{p}+I_{s}}
$$

The estimation of the rotation angle is illustrated in Fig. 3. The polarity of the rotation angle indicates the spatial relation between the beam and the discharge path.

\subsection{Detection of shock waves}

Lightning discharge generates shock waves, which accompany variations in the air density and cause fluctuations of the propagating beam. Signals due to the shock waves are shown in Fig. 4. The discharge gap between the needle terminals was $77 \mathrm{~cm}$ and the charge voltage was $-1200 \mathrm{kV}$. The propagating beam passed $4 \mathrm{~cm}$ below and $3 \mathrm{~cm}$ to the left of the high voltage needle electrode. In this case, the rotation angle was not detected because of the spatial separation between the discharge path and the beam. The air density variation accompanying the shock wave does not contribute to the Faraday effect, so the differential output is zero. In Fig. 4, the shock wave appeared $30 \mu$ s after the discharge trigger, so the distance between the discharge path and the propagating beam was calculated as $1 \mathrm{~cm}$. In the experiment, we confirmed that the shock wave could be detected at a few hundred $\mu$ s after the discharge trigger. Therefore, the shock wave signal

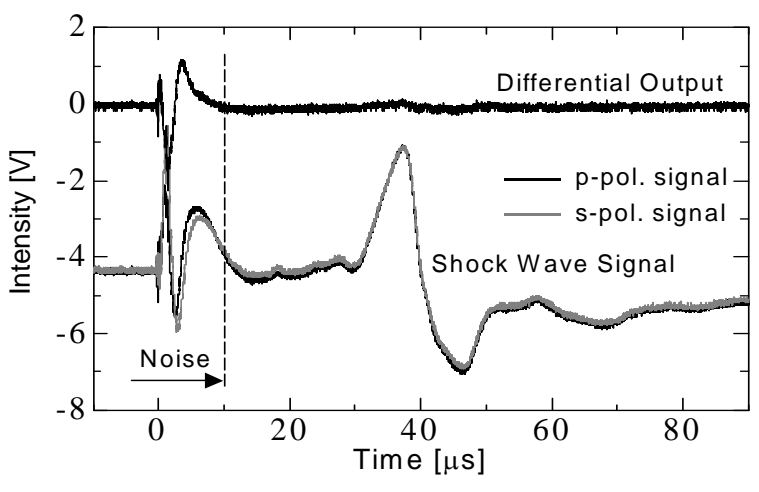

Fig.4 Detection of shock wave.

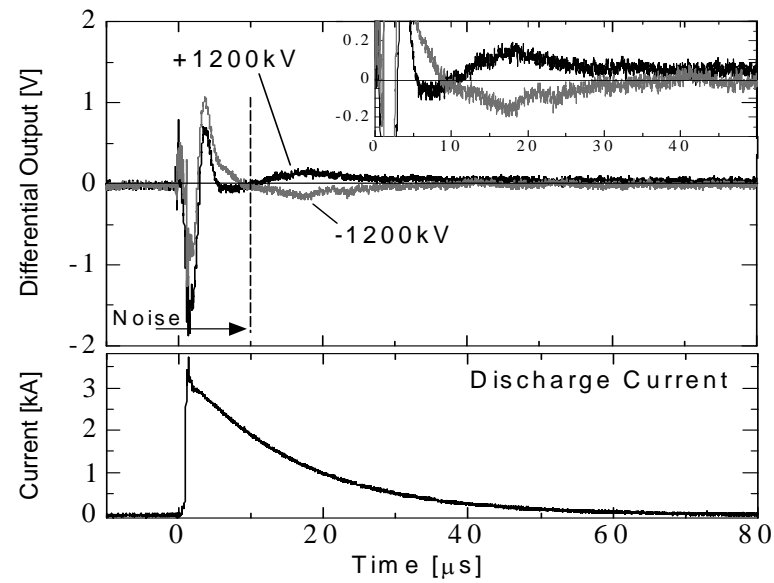

Fig.5 Obtained waveform showing rotation angle detection for positive and negative polarities.

can be used an indicator to locate the discharge location.

\subsection{Detection of polarization rotation angle}

The differential output signals corresponding to the rotation angle of the polarization plane are shown in Fig.5. The typical discharge current is also shown. The discharge gap between the needle terminals was $77 \mathrm{~cm}$ and the charging voltage was $+/-1200 \mathrm{kV}$. The propagating beam passed $2 \mathrm{~cm}$ under the high voltage needle electrode. The waveform before $10 \mu \mathrm{s}$ could not be evaluated because of the electromagnetic noise due to the discharge. The differential outputs in the case of positive discharge $(+1200 \mathrm{kV})$ and negative discharge (-1200 $\mathrm{kV}$ ) showed opposite polarity. The output signals had the same response time as the discharge current. The rotation angle evaluated using eq. (3) was $\delta=0.53$ degrees for positive polarity and $\delta=0.50$ degrees for negative polarity. The dynamic range of $>30 \mathrm{~dB}$ of the differential amplifier enabled detection of the small rotation angle. The results were in agreement with the results of numerical analysis and preliminary experiment using short gap discharge.

To suppress the electromagnetic noise, the receiver optics and electrical circuits were put in a shielded room. Due to spatial limitations caused by the introduction of the shielded room, the position of the propagating beam was changed to 30 
(a)Differential outputs of rotation angles
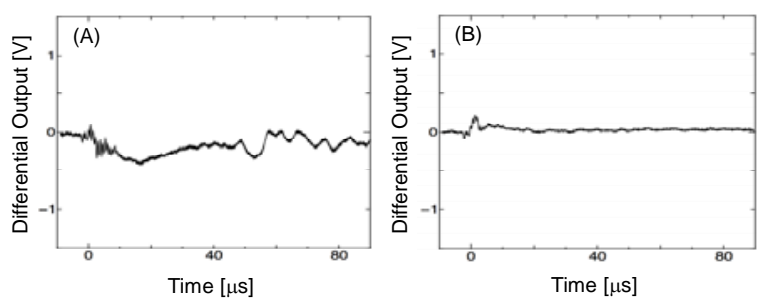

(b)Snapshots of discharge path
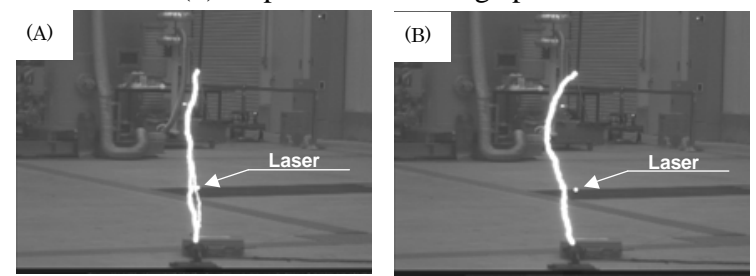

Fig.6 Discharge experiment with electromagnetic shield room.

$\mathrm{cm}$ above the ground needle electrode from $2 \mathrm{~cm}$ below the high voltage needle electrode. In arc or spark discharges, the electron density does not vary significantly throughout the discharge path. The discharge gap length was extended from $77 \mathrm{~cm}$ to $100 \mathrm{~cm}$. This caused the shot-to-shot fluctuations of the discharge path in the extended discharge gap. The rotation angle depends on the distance between the discharge path and the propagating beam. Figure 6 shows the results of the experiment. The discharge gap between the needle terminals was $100 \mathrm{~cm}$ and the charge voltages were $+1200 \mathrm{kV}$. Fig. 6(a) shows the differential output signals. The influence of the electromagnetic noise on the waveform decreased in comparison with the former experiment. Photographs of the discharge path in Fig. 6(b) were obtained simultaneously with the waveforms in Fig. 6(a). The position of the propagating laser beam is also indicated. The separation distance between the beam center and the discharge path was $<2 \mathrm{~cm}$ for $(\mathrm{A})$ and $>6 \mathrm{~cm}$ for (B). The rotation angle was estimated as 0.54 degrees in case (A). The existence of the differential output is dependent on the distance between the discharge path and the beam. The output signal thus appeared when the probing beam was located within $2 \mathrm{~cm}$ apart from the discharge path, where the atmosphere was nearly perfectly ionized $\left(n_{e} \sim 10^{25} \mathrm{~m}^{-3}\right)$.

The present sensitivity of the rotation angle of the polarization plane is $<1$ degree. It is sufficient to detect the rotation angle only in a perfectly ionized atmosphere $\left(n_{e} \sim 10^{25}\right.$ $\left.\mathrm{m}^{-3}\right)$. The rotation angle can be detected only if the transmitting beam crosses the neighborhood of the discharge path. On the other hand, the shock wave does not rotate the polarization plane, and can be detected over a broader spatial region. Therefore, the observation algorithm for lidar application is designed as follows. At first, the lidar system roughly scans the observation region. When a shock wave is detected, the lightning position is estimated. Next, the neighborhood of the lightning position is scanned with higher spatial resolution, and the rotation angle of the polarization plane is measured. The discharge current, magnetic flux density, and ionized density of atmosphere are estimated. The distribution of the ionized atmosphere and its change will lead to the prediction of lightning strike.

\section{Summary}

High-voltage discharge experiments were conducted to detect the small rotation angle of the polarization plane of a propagating beam. The discharge gap length, discharge current, and the spatial arrangement between the discharge path and the propagating beam were changed to examine the influence of the Faraday effect. The estimated rotation angles were less than 1 degree in various conditions. They could be detected by a differential amplifier with a dynamic range of $>30 \mathrm{~dB}$. The experimental results agreed with the results of the numerical analysis and the former experiments using a short gap discharge.

Electromagnetic measurement using the Faraday effect can be applied to lidar measurement of lightning discharges. As the pulse beam enables time-resolved detection, evaluation of the lightning strike position, measurement of the change in the electromagnetic field and spatial distribution of ionization should be possible. Now, a new lidar system is developed for lightning discharge detection. The optics setup is the same as the discharge experiment. The in-line optics, which was a common optics for the transmitter and the receiver, is installed into the system. It takes an advantage to the low-altitude cloud measurement with a narrow field of view. ${ }^{9-11)}$ It is essential to detect a small rotation angle because of the elimination of depolarization caused by multiple scattering.

\section{References}

1) V. Gavrilenko, K. Muraoka, and M. Maeda: Jpn. J. Appl. Phys. 39 (2000) 6455.

2) Y. T. Lin, M. A. Uman, and R. B. Standler: J. Geophys. Res. 85 (1980) 1571.

3) M. J. Master, M. A. Uman, Y. T. Lin, and R. B. Standler: J. Geophys. Res. 86 (1981) 12.127.

4) M. A. Uman: J. Geophys. Res. 90 (1985) 6121.

5) T. Shiina, T. Honda, and T. Fukuchi: IEEJ, A 127 (2007) 187-192 (in Japanese).

6) T. Shiina, T. Honda, and T. Fukuchi: Advanced in Geosciences, submitted.

7) F.Simonet: Rev. Sci. Instrum. 56 (1985) 664-669.

8) K. Kawahata and S. Okajima: Jpn. Soc. Plasma Sci. and Nuclear Fusion Res. 76 (2000) 845 (written in Japanese).

9) T. Shiina, K. Yoshida, M. Ito, and Y. Okamura: Appl. Opt. 44 (2005) 7407.

10) T. Shiina, K. Yoshida, M. Ito, and Y. Okamura: Appl. Opt. 44 (2005) 7467

11) T. Shiina, E. Minami, M. Ito, and Y. Okamura: Appl. Opt. 41 (2002) 3900. 